\title{
AUDIT \\ Lithium monitoring in hospital and general practice
}

\author{
Ann Ryman
}

\begin{abstract}
A survey of the current practice of lithlum monitoring in a general hospital and the general practices it serves revealed erratic and infrequent assessments of serum lithium levels and also of renal and thyroid function. General practitioners, who in this study monitor approximately a third of the patients on lithium, performed worse than psychiatrists, but in nelther case was there cause for complacency.
\end{abstract}

Lithium is a drug widely used in psychiatric practice for the treatment and prophylaxis of bipolar and depressive disorders. Due to its narrow therapeutic index with serious toxic potential at high levels and individual variation in dosage requirements, it is a drug which requires regular serum monitoring. Guidelines have varied over the years. The British National Formulary (BNF; British Medical Association \& Royal Pharmaceutical Society, 1995) currently recommends monitoring serum lithium concentrations at three-monthly intervals in stabilised regimes, aiming for plasma concentrations between 0.4 and $1.0 \mathrm{mmol} / \mathrm{l}$.

Lithium may have toxic effects on renal function, and even at therapeutic concentrations may cause histological and functional changes in the kidney. Compromised renal function reduces the clearance of lithium. It is therefore important that this is monitored, particularly before commencing treatment, at times of physical illness or in the elderly. Thyroid function may also be compromised, and 'regular' monitoring is recommended by the BNF. Six-monthly monitoring of urea and electrolytes and thyroid function is generally accepted as sufficient in stable regimes without complications (Gelder et al, 1996).

There is evidence that these guidelines are often not met and that substandard monitoring of lithium therapy may be widespread (Kehoe \& Mander, 1992). This study aimed to assess the current practice of lithium monitoring in hospital and general practice in Gateshead, and to investigate the frequency of renal and thyroid assessments.

\section{The study}

The database of the biochemistry laboratory at the Queen Elizabeth Hospital, Gateshead, was used to identify all patients undergoing serum lithium estimation in 1994 and 1995. This hospital serves the metropolitan borough of Gateshead with a population of just over 200000.

Patients for whom there were insufficient data (less than three lithium estimations recorded) or for whom the course of treatment was less than three months were excluded. The remaining patients were assumed to be taking lithium regularly for at least three months during the period of analysis. Lithium estimations taken while stabilising the patient on lithium, adjusting the dose, during in-patient stay or physical illness, or following a high reading were recorded separately. At all other times the subjects were assumed to be on a stable lithium regime.

The BNF recommendations for frequency of monitoring and lithium levels were taken as the gold standard. The proportion of patients meeting the standards for frequency throughout the twoyear period were recorded, as well as those in whom they were exceeded at least once. Lithium levels outwith the therapeutic range, taken as being below 0.4 or above $1.0 \mathrm{mmol} / 1$, and any actions taken in the form of more frequent monitoring, were noted. The frequency of assessment of biochemical profiles including urea and electrolyte concentrations and thyroid function tests were also recorded. The number of subjects meeting 'ideal' monitoring criteria (i.e. at least three-monthly lithium assessments, six-monthly urea and electrolyte and thyroid assessments, and lithium levels within the recommended range of action taken if outside these limits) were documented.

The information was analysed descriptively as a whole, and subjects monitored by psychiatrists and general practioners (GPs) were compared.

\section{Results}

\section{Subjects}

Four-hundred and nineteen subjects had had lithium estimations in the 24-month period; 149 (35.6\%) subjects were excluded, 128 (32\%) because of insufficient data and 21 (3.6\%) as being non-lithium users, having only a single 
Table 1. Intervals between lithium assays

\begin{tabular}{lccc}
\hline & All subjects & Psychiatrists & GPs \\
\hline Maximum delay & 52 weeks & 52 weeks & 39 weeks \\
Intervals & & & \\
all $<13$ weeks & $90(33.3 \%)$ & $65(33.3 \%)$ & $19(29.7 \%)$ \\
all $<26$ weeks & $206(76.3 \%)$ & $147(75.4 \%)$ & $46(71.9 \%)$ \\
all least one $>26$ weeks & $64(23.7 \%)$ & $48(24.6 \%)$ & $18(28.1 \%)$ \\
\hline
\end{tabular}

Table 2. High lithium levels

\begin{tabular}{llll}
\hline & All subjects & Psychiatrists & GPs \\
\hline Lithium level $>1.0 \mathrm{mmol} / \mathrm{l}$ & 69 & 42 & 22 \\
No repeat level within 3 weeks (\%) & $43(62.3 \%)$ & $22(52.4 \%)$ & $19(86.4 \%)$ \\
\hline
\end{tabular}

sample with a level of $<0.1 \mathrm{mmol} / 1$ or having only taken the lithium in overdose.

Two hundred and seventy subjects were identified for full analysis, having been stable on lithium for more than three months of the two-year period. Of these, $64(23.7 \%)$ were monitored by GPs, $195(72.2 \%)$ by psychiatrists and $11(4.1 \%)$ by both. This latter group were included in the 'all subjects' analysis, but were excluded when comparing psychiatrists' and GPs' performances separately. The subjects' ages ranged from 19 to 94 years, with a mean age of 56.3 years. There was no significant difference between the ages of subjects monitored by GPs or psychiatrists.

\section{Lithium monitoring}

The variation in monitoring practice was large, with many examples falling well outside the guidelines, and in individual patients, there was clearly an irregular pattern of monitoring, often on an ad hoc basis. Worryingly, in a large proportion of cases, assessments were not taken until after an interval of more than 13 weeks, and a significant number after 26 weeks (Table 1).

The number of occasions where a routine lithium estimation was more than $1.0 \mathrm{mmol} / 1$ are recorded (Table 2). Levels as high as $2.5 \mathrm{mmol} / 1$ occurred (excluding lithium levels following overdose). In cases where a high lithium level was returned, only $55.3 \%$ were followed up by a repeat level within three weeks. The actual level did not appear to influence whether a followup sample was taken or not.

In $23(8.5 \%)$ subjects, the lithium level was consistently low and likely to be sub-therapeutic.

\section{Renal and thyroid function}

Monitoring of renal and thyroid function followed a similar trend to lithium monitoring, with many cases where monitoring was clearly inadequate or absent (Tables 3 and 4). The proportion of subjects having no estimation of renal or thyroid function was higher in the GP group (Table 4).

\section{Subjects meeting 'ideal' monitoring criteria}

Only 41 (15.2\%) of the 270 subjects identified for full analysis met these standards in all areas. Thirty-four (17.4\%) subjects monitored by psychiatrists and $7(10.9 \%)$ of those monitored by GPs fulfilled all the criteria.

Table 3. Frequency of renal and thyroid monitoring of patients

\begin{tabular}{llll}
\hline & $<6$-monthly & $<12$-monthly & $>12$-monthly \\
\hline Urea and electrolyte & $180(66.6 \%)$ & $212(78.5 \%)$ & $27(10 \%)$ \\
Thyroid function tests & $128(47.4 \%)$ & $207(76.7 \%)$ & $33(12.2 \%)$ \\
\hline
\end{tabular}

Table 4. Number of patients with an absence of renal and thyroid monitoring

\begin{tabular}{llll}
\hline & All subjects & Psychiatrist & GPs \\
\hline Urea and electrolyte - no result & $31(11.5 \%)$ & $14(7 \%)$ & $17(26.6 \%)$ \\
Thyroid function tests - no result & $30(11.1 \%)$ & $9(4.6 \%)$ & $20(31 \%)$ \\
\hline
\end{tabular}




\section{Comment}

There is cause for concern in the wide variations in practice within and between patients and the high proportion of cases where monitoring is at best less than adequate. This applies to both monitoring of serum lithium levels and also to renal and thyroid function. Only a third of patients had been adequately monitored throughout the two-year period, according to the guidelines accepted for this study. Even if more lenient guidelines were used, a quarter of subjects had not had a lithium estimation for over six months at least once during the study period, and a fifth had had renal and thyroid function assessed less than annually, half of these not having had an assessment at all for two years.

In two-thirds of cases where a high lithium level was returned, no action was taken, with worrying potential consequences. Although GPs performed worse than psychiatrists, this also applies to half of those cases monitored by psychiatrists.

A number of subjects had consistently subtherapeutic levels of lithium. Although lower doses are recommended in the elderly, the levels should still be within the therapeutic range. In a small proportion of cases it is possible that there was evidence of clinical efficacy despite an apparently low dose, but the long-term risks of taking a potentially toxic drug in a sub-therapeutic dose are self-evident.

Subjects for whom only one or two estimations of lithium were recorded in the two-year period were excluded from the main analysis, but form a significant group. These may be due to serious deficits in monitoring, a cause of grave concern. or because the subject received only a short course of lithium. The latter may be due to inefficacy, poor tolerance, or lack of compliance. Abrupt cessation of lithium therapy or short courses in bipolar disorder have been shown to precipitate or advance the next episode (Goodwin, 1994). Careful selection of suitably compliant patients is therefore necessary to avoid inducing an iatrogenic episode.

The results are in keeping with previous studies in this area (Kehoe \& Mander, 1992), suggesting that substandard monitoring of lithium therapy may be widespread. The Medical Protection Society has reported that $10 \%$ of claims for psychiatric negligence involve inadequate mon- itoring of lithium treatment (Medical Protection Society, 1989). This is therefore an area of great importance in improving psychiatric care.

\section{Recommendations}

Standardisation of practice to within recommended guidelines by:

(a) better communication between GPs and psychiatrists as to who is responsible for monitoring:

(b) introduction of locally agreed standards of monitoring practice including renal and thyroid monitoring, and action to be taken in the event of a potentially toxic or subtherapeutic result;

(c) feedback on current performance and education of GPs and senior house officers as to recommended guidelines;

(d) consideration to be given to the setting up of a lithium register or clinic; and

(e) complete the audit cycle by repeating the study after implementation of these changes.

\section{Acknowledgements}

I thank Dr B. Browne (Consultant Psychiatrist) and Dr D. Peel, Dr G. Handley and Mr E. Why of the Biochemistry Department, Queen Elizabeth Hospital Gateshead, for their support in obtaining the data for this study.

\section{References}

Brtish Medical association \& Royal Pharmaceutical SOCIETY (1995) British National Formulary. 30, 164-166.

Gelder, M., GATH, D., MAYOU, R., et al (1996) Oxford Textbook of Psychiatry (3rd edn). Oxford: Oxford University Press.

GoodwIN, G. M. (1994) Recurrence of mania after lithium withdrawal. Implications for the use of lithium in the treatment of bipolar affective disorder. British Journal of Psychiatry, 164, 149-152.

KEHOE, R. F. \& MANDER, A. J. (1992) Lithium treatment: prescribing and monitoring habits in hospital and general practice. British Medical Journal. S04. 552-554. MEDICAL PROTECTION SOCIETY (1989) Psychiatric negligence. Medical Notes, 1. 2.

Ann Ryman, Tranwell Unit, Queen Elizabeth Hospital, Gateshead 